\title{
Rhodium(III)-Catalyzed Addition of Indoles with Boc-Imines via C-H Bond Activation
}

\author{
Jinfang Wang, ${ }^{*, a}$ Hui Wang ${ }^{b}$ and Chuanjun Yue ${ }^{a}$ \\ ${ }^{a}$ Changzhou Institute of Technology, 666 Liao He Road, 213032 Changzhou, Jiangsu, China \\ ${ }^{b}$ Changzhou Le Sun Pharmaceuticals Ltd., 518 Yun He Road, 213125 Changzhou, Jiangsu, China
}

\begin{abstract}
A rhodium-catalyzed alkylation reaction of indoles with $N$-Boc-imines has been developed via $\mathrm{C}-\mathrm{H}$ activation to afford a series of substituted 2-indolyl-methanamine derivatives with good functional group tolerance and regioselectivity. A wide range of indole-based alkylation products could be obtained in up to $95 \%$ yield.
\end{abstract}

Keywords: rhodium(III)-catalysis, $\mathrm{C}-\mathrm{H}$ activation, indole

\section{Introduction}

Transition-metal-catalyzed aromatic $\mathrm{C}-\mathrm{H}$ functionalization has been recognized to be a highly important synthetic tool for its atom-economical routes to functionalized aromatic molecules. ${ }^{1}$ Recently, it has been demonstrated that $\mathrm{Rh}^{\mathrm{III}}$ catalysts are highly efficient for the activation of $\mathrm{sp}^{2} \mathrm{C}-\mathrm{H}$ bonds of aromatic compounds in the coupling with unsaturated molecules and with electrophilic reagents. ${ }^{2}$ Particularly, $\mathrm{Rh}^{\mathrm{III}}$ catalytic $\mathrm{C}-\mathrm{H}$ bond activation is an attractive strategy for preparing amino-containing aromatic compounds which are commonly found in pharmaceuticals as well as natural products and functional materials. ${ }^{3}$

Indoles derivatives are of great interest in organic synthesis because of their presence in numerous natural products and pharmaceuticals. ${ }^{4}$ Among them, 2-indolylmethanamine derivatives are particularly important because of their ubiquitous presence in numerous biologically active compounds. ${ }^{5-9}$ Therefore, methods for a general, rapid, and regioselective preparation of 2-indolyl-methanamines would be highly desirable. Zhou et al..$^{10}$ successfully reported a $\mathrm{Rh}^{\mathrm{III}}$-catalyzed regioselective addition of indole $\mathrm{C}-\mathrm{H}$ bonds to aryl- and alkyl- $N$-sulfonylimines for the preparation of 2-indolyl-methanamine derivatives with good functional group tolerance, but in relatively low yields (between 42 to $71 \%$, see Scheme 1). Due to the importance of 2-indolyl methanamine derivatives, herein we report a rhodium(III)catalyzed direct and selective C-2 alkylation reaction of indoles with $\mathrm{N}$-Boc-imines via $\mathrm{C}-\mathrm{H}$ activation, affording a

*e-mail: wangjf1001@126.com series of substituted 2-indolyl-methanamine derivatives with good functional group tolerance and in high yields under mild conditions. These compounds are potential building blocks for preparing biologically active compounds.

\section{Results and Discussion}

To explore the optimum reaction conditions, 1-( $N, N$-dimethylcarbamoyl) indole (1a) and benzaldehyde $N$-(tert-butoxycarbonyl)imine (2a) were chosen as model substrates for the synthesis of 2-indolylmethanamine derivative (3a) (Table 1). Initially, we applied $\left[\mathrm{RhCp} * \mathrm{Cl}_{2}\right]_{2}$ $(5 \mathrm{~mol} \%)$ as a catalyst without any additive. However, no desired product was detected after $6 \mathrm{~h}$, and $\mathbf{1 a}$ was mostly recovered (Table 1, entry 1 ). Then we chose $\mathrm{AgCO}_{2} \mathrm{CF}_{3}$ as an additive and partial conversion of $1 \mathrm{a}$ was observed after $6 \mathrm{~h}$ at $75^{\circ} \mathrm{C}$ with a low yield of $30 \%$ (Table 1, entry 2). Replacement of $\mathrm{AgCO}_{2} \mathrm{CF}_{3}$ with $\mathrm{AgSO}_{3} \mathrm{CF}_{3}$ did not give a satisfactory result (Table 1, entry 3 ) too. To our delight, switching the additive to $\mathrm{AgSbF}_{6}$ gave rise to an efficient coupling with up to $85 \%$ isolated yield (Table 1, entry 4). When the reaction was not performed under an inert nitrogen atmosphere, the yield decreased sharply from 85 to $34 \%$ (Table 1, entry 5). Strong solvent effects have also been observed, such as the reaction proceeded well in 1,2-dichloroethane (DCE) and dichloromethane (DCM), whereas toluene (PhMe) and tert-butyl alcohol $(t-\mathrm{BuOH})$ were not suitable for this reaction, in which we could not observe the full conversion of starting materials despite prolonging the reaction time to $24 \mathrm{~h}$ (Table 1, entries 6-8). Reducing the amount of either catalyst or additive led to decreased yields (Table 1, entries 9-10). 
Previous work: Zhou et al., 2013 (adapted)<smiles></smiles><smiles>[R]NC([R2])c1cc2c[Y10]([H])ccc2n1[R1]</smiles>

moderate yields

long reaction time<smiles></smiles>

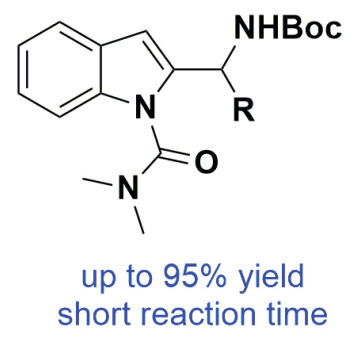

Scheme 1. Synthetic strategies to produce 2-indolyl-methanamines.

Further investigations revealed that the reaction could occur at a milder temperature (Table 1, entry 11). When the temperature was raised to $50{ }^{\circ} \mathrm{C}$, the yield dropped down to $68 \%$ (Table 1, entry 12) and many side products were detected. Altogether, the optimal result was obtained by treating $1 \mathbf{a}$ and 1.5 equivalent of $2 \mathbf{a}$ in DCE using catalyst
$\left[\mathrm{RhCp} * \mathrm{Cl}_{2}\right]_{2}(5 \mathrm{~mol} \%)$ and additive $\mathrm{AgSbF}_{6}(20 \mathrm{~mol} \%)$ at $75{ }^{\circ} \mathrm{C}$ for $6 \mathrm{~h}$ under an inert nitrogen atmosphere.

Under the aforementioned optimized reaction condition, we examined the substrate scope of this reaction with various substituted 1 -( $N, N$-dimethylcarbamoyl) indoles and imines (Table 2). In general, a range of substituted

Table 1. Optimization of the Rh-catalyzed direct addition reaction ${ }^{\mathrm{a}}$<smiles>CN(C)C(=O)n1ccc2ccccc21</smiles><smiles>O=[N+](O)/C=C\c1ccccc1</smiles>

$\left[\mathrm{Cp}^{*} \mathrm{RhCl}_{2}\right]_{2} /$ additive solvent $/ \mathrm{T}{ }^{\circ} \mathrm{C} / 6 \mathrm{~h}$<smiles>CN(C)C(=O)n1c(C(NC(C)(C)C)c2ccccc2)cc2ccccc21</smiles>

3a

\begin{tabular}{|c|c|c|c|c|c|c|}
\hline entry & Catalyst & Solvent & Additive & Temperature $/{ }^{\circ} \mathrm{C}$ & time / h & Yield $/ \%$ \\
\hline 1 & {$\left[\mathrm{Cp} * \mathrm{RhCl}_{2}\right]_{2}$} & DCE & - & 75 & 6 & 0 \\
\hline 2 & {$\left[\mathrm{Cp} * \mathrm{RhCl}_{2}\right]_{2}$} & DCE & $\mathrm{AgCO}_{2} \mathrm{CF}_{3}$ & 75 & 6 & 30 \\
\hline 3 & {$\left[\mathrm{Cp} * \mathrm{RhCl}_{2}\right]_{2}$} & DCE & $\mathrm{AgSO}_{3} \mathrm{CF}_{3}$ & 75 & 6 & 37 \\
\hline 4 & {$\left[\mathrm{Cp} * \mathrm{RhCl}_{2}\right]_{2}$} & DCE & $\mathrm{AgSbF}_{6}$ & 75 & 6 & 85 \\
\hline $5^{\mathrm{c}}$ & {$\left[\mathrm{Cp} * \mathrm{RhCl}_{2}\right]_{2}$} & DCE & $\mathrm{AgSbF}_{6}$ & 75 & 6 & 34 \\
\hline 6 & {$\left[\mathrm{Cp} * \mathrm{RhCl}_{2}\right]_{2}$} & $\mathrm{PhMe}$ & $\mathrm{AgSbF}_{6}$ & 75 & 24 & 61 \\
\hline 7 & {$\left[\mathrm{Cp} * \mathrm{RhCl}_{2}\right]_{2}$} & $t$-BuOH & $\mathrm{AgSbF}_{6}$ & 75 & 24 & 23 \\
\hline 8 & {$\left[\mathrm{Cp} * \mathrm{RhCl}_{2}\right]_{2}$} & DCM & $\mathrm{AgSbF}_{6}$ & 75 & 6 & 71 \\
\hline $9^{d}$ & {$\left[\mathrm{Cp} * \mathrm{RhCl}_{2}\right]_{2}$} & DCE & $\mathrm{AgSbF}_{6}$ & 75 & 6 & 47 \\
\hline $10^{\mathrm{e}}$ & {$\left[\mathrm{Cp} * \mathrm{RhCl}_{2}\right]_{2}$} & DCE & $\mathrm{AgSbF}_{6}$ & 75 & 6 & 62 \\
\hline 11 & {$\left[\mathrm{Cp} * \mathrm{RhCl}_{2}\right]_{2}$} & DCE & $\mathrm{AgSbF}_{6}$ & 25 & 6 & 72 \\
\hline 12 & {$\left[\mathrm{Cp} * \mathrm{RhCl}_{2}\right]_{2}$} & DCE & $\mathrm{AgSbF}_{6}$ & 50 & 6 & 68 \\
\hline
\end{tabular}

a Reaction conditions: 1a $(0.10 \mathrm{mmol})$, $2 \mathrm{a}(0.15 \mathrm{mmol})$, catalyst $(5 \mathrm{~mol} \%)$, additive $(20 \mathrm{~mol} \%)$, and solvent $(1.0 \mathrm{~mL})$ at $75^{\circ} \mathrm{C}$ for $6 \mathrm{~h}$ under nitrogen; ${ }^{\mathrm{b} y i e l d}$ of isolated product; "the reaction was carried out under air; ${ }^{\mathrm{d}}$ the amount of catalyst was decreased to $2.5 \mathrm{~mol} \%$; ${ }^{\mathrm{e}}$ the amount of additive was decreased to $10 \mathrm{~mol} \%$; DCE: 1,2-dichloroethane; PhMe: toluene; $t$-BuOH: tert-butyl alcohol; DCM: dichloromethane. 
Table 2. Substrate scope for the $\mathrm{Rh}^{\mathrm{III}}$-catalyzed addition reaction ${ }^{\mathrm{a}, \mathrm{b}}$

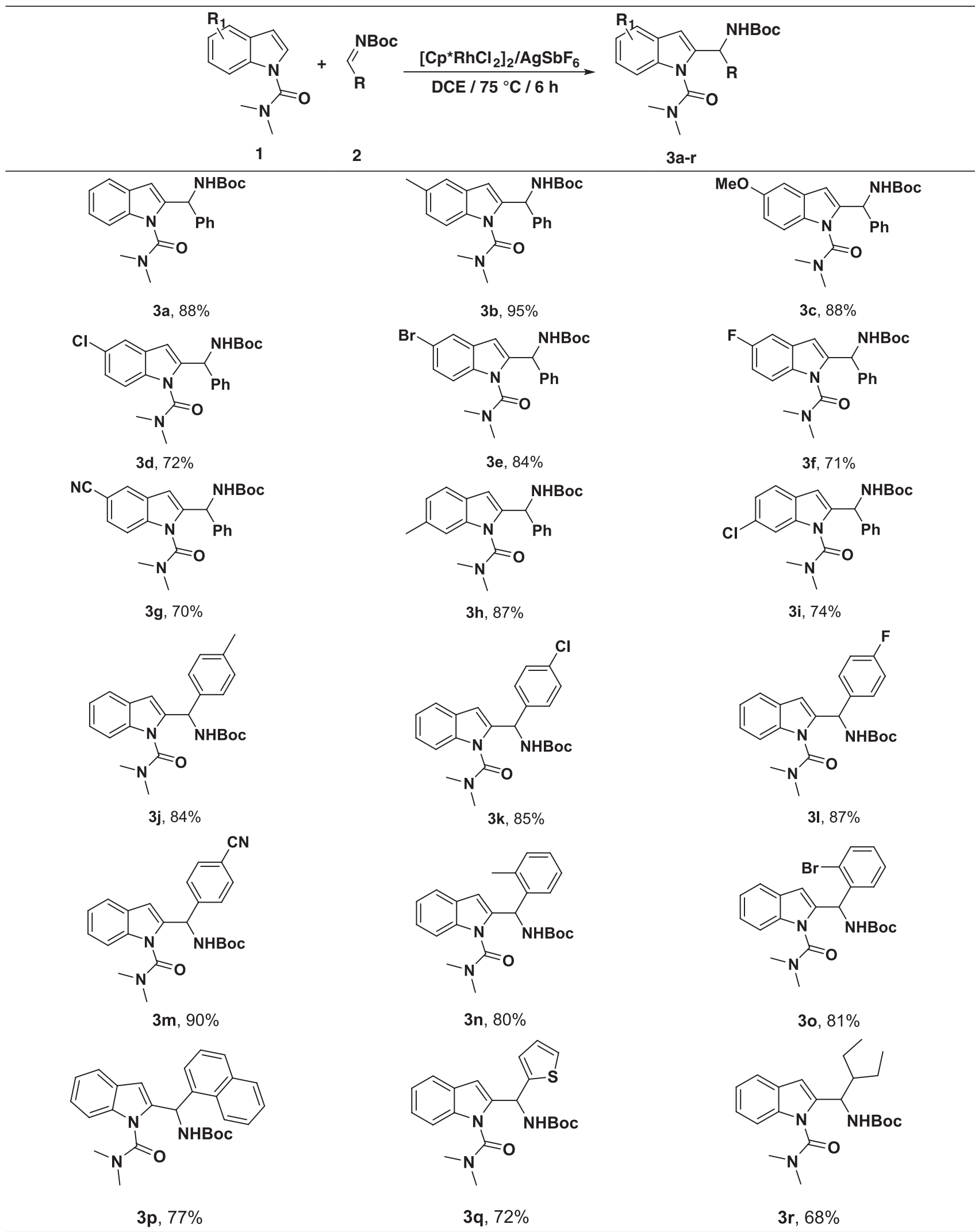

a Reaction conditions: 1a $(0.20 \mathrm{mmol}), \mathbf{2 a}(0.30 \mathrm{mmol})$, catalyst $(5 \mathrm{~mol} \%)$, additive $(20 \mathrm{~mol} \%)$, and solvent $(2.0 \mathrm{~mL})$ at $75^{\circ} \mathrm{C}$ for $6 \mathrm{~h}$ under nitrogen; ${ }^{\text {byield }}$ of isolated product. 
1-(N,N-dimethylcarbamoyl) indole derivatives and imines with electron-withdrawing or electron-donating groups were all successfully transformed into the corresponding adducts in good to excellent yields $(68-95 \%, \mathbf{3 a - 3 0})$. Introduction of electron-donating groups (-Me, and $-\mathrm{OMe})$ to the indole ring gave high yields $(\mathbf{3 b}$ and $\mathbf{3 c})$. The chloro (3d) and bromo (3e) atoms were highly compatible with this addition reaction. Tolerance to the chloro and bromo is especially noteworthy since they are useful for subsequent cross-coupling reactions. However, the substrates substituted by a fluoro and cyano moiety at the 5-position of the indole resulted in slightly decreased yields, respectively (3f and $\mathbf{3 g}$ ). Substitution with methyl or chloro at 6-position of indole also gave good yields $(\mathbf{3 h}$ and $\mathbf{3 i}$ ). The results also indicated that the electronic property of the substitutes and the positions of substitution on the benzene ring of imines have no significant influence on the yields of these adducts (3j-3o). Nathphyl, thiofuran and 3-amyl groups were also introduced to the imines and good yields were obtained (3p-3r).

Furthermore, a gram-scale reaction was conducted to evaluate the reaction efficacy on a preparative scale. The reaction of 1-( $N, N$-dimethylcarbamoyl)indole (1a) with benzaldehyde $N$-(tert-butoxycarbonyl)imine (2a) under the standard conditions provided the target product in $87 \%$ yield (Scheme 2). Therefore, the present method is very effective for the synthesis of $\mathbf{3 a}$.

Based on the previous work, ${ }^{3,11,12}$ we proposed the following mechanism (Scheme 3). First, an active catalyst is generated through anion exchange with $\mathrm{AgSbF}_{6}$, then a $\mathrm{N}, \mathrm{N}$-dimethylcarbamoyl-directed $\mathrm{C}-\mathrm{H}$ bond activation occur through deprotonation-metalation to give $\mathbf{A}$, coordination of the $N$-Boc-imine $(\mathbf{B})$ would then activate the imine for migratory insertion to form the $N$-Rh species $\mathbf{C}$. In the last step, $\mathbf{C}$ is protonated to provide the desired indole derivative 3 accompanied by the regeneration of the active catalyst.

\section{Conclusions}

In summary, we have developed an efficient methodology for the addition of 1-(N,N-dimethylcarbamoyl) indoles with tert-butyloxy carbonyl protected imines via $\mathrm{Rh}^{\mathrm{III}}$-catalyzed $\mathrm{C}-\mathrm{H}$ activation reaction to afford biologically relevant 2-indolylmethanamines with good functional group tolerance and selectivity in good to excellent yields. In view of the potential use of these 2-indolylmethanamines, we expect this method to be widely used in the pharmaceutical field.

\section{Experimental}

\section{General information}

The analytical thin layer chromatography (TLC) used was HSGF 254 (0.15-0.2 mm thickness). All products were characterized by their nuclear magnetic resonance (NMR) and mass spectrometry (MS) spectra. ${ }^{1} \mathrm{H}$ and ${ }^{13} \mathrm{C}$ NMR spectra were recorded on a 400 or $500 \mathrm{MHz}$ instrument using tetramethylsilane as internal reference. Data are presented as follows: chemical shift, multiplicity ( $\mathrm{s}=$ singlet, br $\mathrm{s}=$ broad singlet, $\mathrm{d}=$ doublet, $\mathrm{br} \mathrm{d}=$ broad doublet, $\mathrm{t}=$ triplet, $\mathrm{m}=$ multiplet $), J=$ coupling constant in hertz (Hz). Silica gel 60H (200-300 mesh) manufactured by Qingdao Haiyang Chemical Group Co. (China) was used for general chromatography. Dichloromethane, 1,2-dichloroethane and toluene were distilled over $\mathrm{CaH}_{2} . \mathrm{AgSbF}_{6}, \mathrm{AgOTf}, \mathrm{AgSO}_{3} \mathrm{CF}_{3}$ and $\left[\mathrm{Cp} * \mathrm{RhCl}_{2}\right]_{2}$ were purchased from Energy Chemical Co. and used without further purification. Substrate 1-(N,N-dimethylcarbamoyl) indoles ${ }^{10}$ and $N$-boc imines ${ }^{13-18}$ were synthesized according to published procedures.

\section{General synthesis procedures of $\mathbf{3 a}$ to $\mathbf{3 r}$}

In a reaction tube, $\left[\mathrm{Cp} * \mathrm{RhCl}_{2}\right]_{2}(0.01 \mathrm{mmol}, 6.2 \mathrm{mg})$, $\mathrm{AgSbF}_{6}(0.04 \mathrm{mmol}, 13.7 \mathrm{mg})$, substrate $\mathbf{1 a}(0.20 \mathrm{mmol}$, 1.0 equiv), and $\mathbf{2 a}(0.30 \mathrm{mmol}, 1.5$ equiv) were added followed by addition of DCE $(2.0 \mathrm{~mL})$. The vessel was sealed and heated at $75{ }^{\circ} \mathrm{C}$ (oil bath temperature) for $6 \mathrm{~h}$ under an inert nitrogen atmosphere. The resulting mixture was cooled to room temperature, filtered through a short silica gel pad and transferred to silica gel column directly to give the product. Following general procedure,

Gram-Scale Synthesis

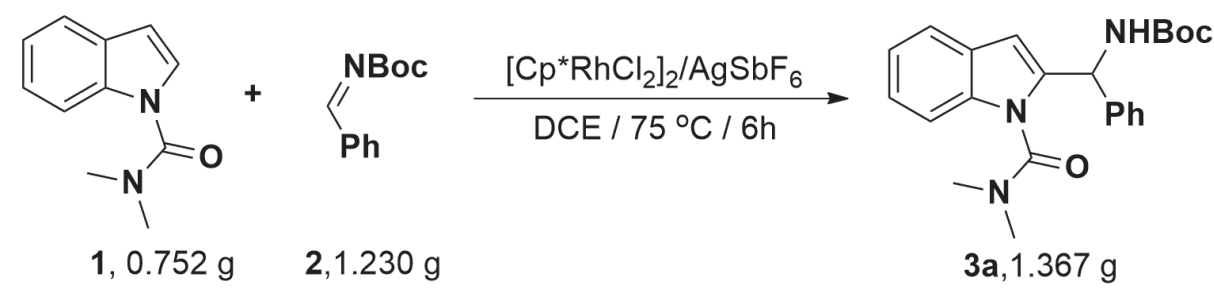

Scheme 2. Gram-scale synthesis. 


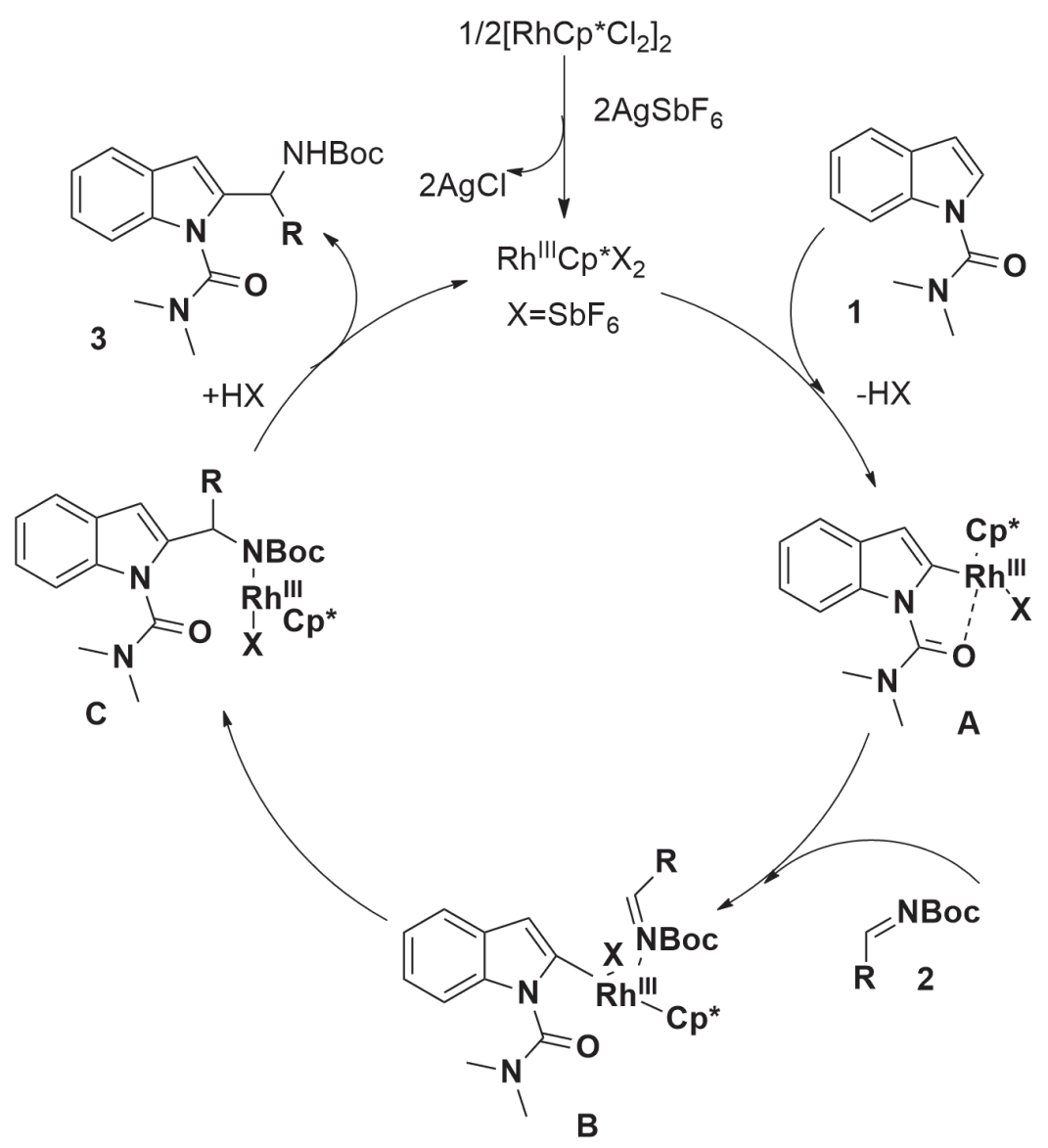

Scheme 3. Proposed catalytic cycle.

compound 3a was purified by column chromatography on silica gel using petroleum ether:ethyl acetate $(5: 1)$ in $85 \%$ isolated yield as an off-white solid.

\section{Analytical characterization data of products}

tert-Butyl((1-(dimethylcarbamoyl)-1H-indol-2-yl)(phenyl) methyl)carbamate (3a)

Following a general procedure: $69 \mathrm{mg}, 88 \%$ yield; white solid; ${ }^{1} \mathrm{H}$ NMR (400 MHz, $\mathrm{CDCl}_{3}$ ) $\delta$ 7.64-7.62 (d, $1 \mathrm{H}, J 8.0 \mathrm{~Hz}, \mathrm{Ar}-\mathrm{H}), 7.47-7.45$ (d, 1H, $J 8.0 \mathrm{~Hz}, \mathrm{Ar}-\mathrm{H})$, 7.39-7.26 (m, 6H, Ar-H, N-H), 7.15 (m,1H, Ar-H), 6.95 (s, 1H, Ar-H), 6.20 (s, Ar-1H), 5.22 (s, 1H, C-H), 3.06 (s, $\left.6 \mathrm{H}, \mathrm{N}\left(\mathrm{CH}_{3}\right)_{2}\right), 1.48\left(\mathrm{~s}, 9 \mathrm{H}, \mathrm{C}\left(\mathrm{CH}_{3}\right)_{3}\right) ;{ }^{13} \mathrm{C} \mathrm{NMR}(100 \mathrm{MHz}$, $\left.\mathrm{CDCl}_{3}\right) \delta 155.10,154.85,136.25,128.58,127.60,127.51$, $126.95,124.93,124.00,121.82,120.84,119.88,113.60$, 79.82, 51.42, 38.44, 28.40; HRMS (ESI) $\mathrm{m} / z$ calcd. for $\mathrm{C}_{23} \mathrm{H}_{28} \mathrm{~N}_{3} \mathrm{O}_{3}{ }^{+}[\mathrm{M}+\mathrm{H}]^{+}$: 394.2125; found: 394.2121 .

tert-Butyl((1-(dimethylcarbamoyl)-5-methyl-1H-indol-2-yl) (phenyl)methyl)carbamate (3b)

Following a general procedure: $77.3 \mathrm{mg}, 95 \%$ yield; white solid; ${ }^{1} \mathrm{H}$ NMR $\left(500 \mathrm{MHz}, \mathrm{CDCl}_{3}\right) \delta 7.51-7.50(\mathrm{~d}, 1 \mathrm{H}$,
$J$ 8.4 Hz, Ar-H), 7.41-7.36 (m, 4H, Ar-H, N-H), 7.33-7.30 (m, 2H, Ar-H), 7.15-7.13 (dd, 1H, J 8.4, 1.1 Hz, Ar-H), 6.87 (s, 1H, Ar-H), $6.18(\mathrm{~d}, J 6.8 \mathrm{~Hz}, 1 \mathrm{H}, \mathrm{Ar}-\mathrm{H}), 5.21(\mathrm{~s}, 1 \mathrm{H}$, $\mathrm{C}-\mathrm{H}), 3.04\left(\mathrm{~s}, 6 \mathrm{H}, \mathrm{N}\left(\mathrm{CH}_{3}\right)_{2}\right), 2.42\left(\mathrm{~s}, 3 \mathrm{H}, \mathrm{CH}_{3}\right), 1.49$ (s, 9H, $\left.\mathrm{C}\left(\mathrm{CH}_{3}\right)_{3}\right) ;{ }^{13} \mathrm{C} \mathrm{NMR}\left(126 \mathrm{MHz}, \mathrm{CDCl}_{3}\right) \delta 155.17,154.98$, $141.07,134.48,131.36,128.56,127.89,127.45,126.91$, $125.50,125.09,119.61,113.31,79.92,51.22,38.45,28.41$, 21.39; HRMS (ESI) $m / z$ calcd. for $\mathrm{C}_{24} \mathrm{H}_{30} \mathrm{~N}_{3} \mathrm{O}_{3}{ }^{+}[\mathrm{M}+\mathrm{H}]^{+}$: 408.2282; found: 408.2280 .

tert-Butyl((1-(dimethylcarbamoyl)-5-methoxy-1 H-indol-2-yl) (phenyl)methyl)carbamate (3c)

Following general procedure: $74.5 \mathrm{mg}, 88 \%$ yield; white solid; ' ${ }^{1} \mathrm{H}$ NMR $\left(400 \mathrm{MHz}, \mathrm{CDCl}_{3}\right) \delta 7.51(\mathrm{~d}, 1 \mathrm{H}$, $J$ 8.7 Hz, Ar-H), 7.38-7.29 (m, 5H, Ar-H, N-H), 6.93-6.85 (m, 3H, Ar-H), 6.15 (s, 1H, Ar-1H), 5.17 (s, 1H, C-H), 3.77 (s, 3H, $\left.\mathrm{CH}_{3}\right), 3.01\left(\mathrm{~s}, 6 \mathrm{H}, \mathrm{N}\left(\mathrm{CH}_{3}\right)_{2}\right), 1.45\left(\mathrm{~s}, 9 \mathrm{H}, \mathrm{C}\left(\mathrm{CH}_{3}\right)_{3}\right)$; ${ }^{13} \mathrm{C}$ NMR $\left(100 \mathrm{MHz}, \mathrm{CDCl}_{3}\right) \delta 155.54,155.33,155.15$, $131.31,128.79,128.58,127.72,127.13,125.69,114.68$, $113.74,102.13,79.75,55.89,51.22,38.68,28.61$; HRMS (ESI) $\mathrm{m} / z$ calcd. for $\mathrm{C}_{24} \mathrm{H}_{30} \mathrm{~N}_{3} \mathrm{O}_{4}{ }^{+}[\mathrm{M}+\mathrm{H}]^{+}:$424.2231; found: 424.2232 . 
tert-Butyl((5-chloro-1-(dimethylcarbamoyl)-1 H-indol-2-yl) (phenyl)methyl)carbamate (3d)

Following general procedure: $61.5 \mathrm{mg}, 72 \%$ yield; white solid; ${ }^{1} \mathrm{H}$ NMR $\left(500 \mathrm{MHz}, \mathrm{CDCl}_{3}\right) \delta 7.66(\mathrm{~s}, 1 \mathrm{H})$, 7.38-7.37 (m, 4H, Ar-H, N-H), 7.34-7.32 (m, 2H, Ar-H), 7.14 (dd, 1H, J 8.4 Hz, $1.5 \mathrm{~Hz}, \mathrm{Ar}-\mathrm{H}), 6.96$ (s, 1H, Ar-H), $6.17(\mathrm{~s}, 1 \mathrm{H}), 5.17(\mathrm{~s}, 1 \mathrm{H}), 3.06\left(\mathrm{~s}, 6 \mathrm{H}, \mathrm{N}\left(\mathrm{CH}_{3}\right)_{2}\right), 1.48(\mathrm{~s}, 9 \mathrm{H}$, $\left.\mathrm{C}\left(\mathrm{CH}_{3}\right)_{3}\right) ;{ }^{13} \mathrm{C} \mathrm{NMR}\left(126 \mathrm{MHz}, \mathrm{CDCl}_{3}\right) \delta 155.07,154.40$, $136.71,130.10,128.70,127.72,126.97,126.06,125.29$, 122.56, 120.71, 113.80, 77.23, 51.29, 38.45, 28.38; HRMS (ESI) $\mathrm{m} / z$ calcd. for $\mathrm{C}_{23} \mathrm{H}_{27} \mathrm{ClN}_{3} \mathrm{O}_{3}{ }^{+}[\mathrm{M}+\mathrm{H}]^{+}: 428.1735$; found: 428.1727 .

tert-Butyl((5-bromo-1-(dimethylcarbamoyl)-1 $\mathrm{H}$-indol-2-yl) (phenyl)methyl)carbamate (3e)

Following general procedure: $79.1 \mathrm{mg}, 84 \%$ yield; white solid; ${ }^{1} \mathrm{H}$ NMR $\left(500 \mathrm{MHz}, \mathrm{CDCl}_{3}\right) \delta 7.60(\mathrm{~s}, 1 \mathrm{H})$, 7.53-7.51 (d, 1H, J 8.8 Hz, Ar-H), 7.41-7.38 (m, 5H, Ar-H), 7.35-7.33 (m, 1H, Ar-H), 6.92 (s, 1H, Ar-H), 6.16 (s, 1H, Ar-H), 5.19 (s, 1H), 3.04 (s, 6H, N( $\left.\left.\mathrm{CH}_{3}\right)_{2}\right), 1.49$ (s, 9H, $\left.\mathrm{C}\left(\mathrm{CH}_{3}\right)_{3}\right) ;{ }^{13} \mathrm{C} \mathrm{NMR}\left(126 \mathrm{MHz}, \mathrm{CDCl}_{3}\right) \delta 155.05,154.37$, $135.02,129.26,128.72,127.74,126.96,126.91,125.88$, $122.57,120.45,115.19,115.12,99.99,80.10,51.30,38.44$, 28.40; HRMS (ESI) $m / z$ calcd. for $\mathrm{C}_{23} \mathrm{H}_{27} \mathrm{BrN}_{3} \mathrm{O}_{3}{ }^{+}[\mathrm{M}+\mathrm{H}]^{+}$: 472.1231; found: 472.1233 .

tert-Butyl((1-(dimethylcarbamoyl)-5-fluoro-1 H-indol-2-yl) (phenyl)methyl)carbamate (3f)

Following general procedure: $58.3 \mathrm{mg}, 71 \%$ yield; white solid; ${ }^{1} \mathrm{H}$ NMR (500 MHz, $\left.\mathrm{CDCl}_{3}\right) \delta$ 7.60-7.59 (s, 1H, Ar-H), 7.38-7.28 (m, 5H, Ar-H, N-H), 7.06-6.99 (m, 3H, Ar-H), 6.14 (s, 1H, Ar-H), 5.20 (s, 1H), 3.06 (s, 6H, $\left.\mathrm{N}\left(\mathrm{CH}_{3}\right)_{2}\right), 1.48\left(\mathrm{~s}, 9 \mathrm{H}, \mathrm{C}\left(\mathrm{CH}_{3}\right)_{3}\right) ;{ }^{13} \mathrm{C} \mathrm{NMR}(126 \mathrm{MHz}$, $\left.\mathrm{CDCl}_{3}\right) \delta 159.58,157.69,155.08,154.65,140.54,132.79$, 128.72 , 127.72, 126.96, 126.22, 114.62, 112.09, 105.36, 79.94, 51.19, 38.47, 28.38; HRMS (ESI) $\mathrm{m} / \mathrm{z}$ calcd. for $\mathrm{C}_{23} \mathrm{H}_{27} \mathrm{FN}_{3} \mathrm{O}_{3}{ }^{+}[\mathrm{M}+\mathrm{H}]^{+}:$412.2031; found: 412.2033 .

tert-Butyl((5-cyano-1-(dimethylcarbamoyl)-1 H-indol-2-yl) (phenyl)methyl)carbamate $(\mathbf{3 g})$

Following general procedure: $58.5 \mathrm{mg}, 70 \%$ yield; white solid; ${ }^{1} \mathrm{H}$ NMR $\left(400 \mathrm{MHz}, \mathrm{CDCl}_{3}\right) \delta 7.72-7.69(\mathrm{~m}$, 2H, Ar-H), 7.53-7.51 (d, 1H, J 8.0 Hz, Ar-H), 7.37-7.33 (m, 5H, Ar-H, N-H), 7.08 (s, 1H, Ar-H), 6.15 (s, 1H), 5.13 (s, 1H), 3.05 (s, 6H, N( $\left.\left(\mathrm{CH}_{3}\right)_{2}\right), 1.46\left(\mathrm{~s}, 9 \mathrm{H}, \mathrm{C}\left(\mathrm{CH}_{3}\right)_{3}\right)$; ${ }^{13} \mathrm{C} \mathrm{NMR}\left(100 \mathrm{MHz}, \mathrm{CDCl}_{3}\right) \delta 155.18,153.98,140.35$, $138.38,129.13,128.27,127.64,127.18,126.82,125.43$, $121.43,119.95,114.75,105.49,80.53,51.67,38.63$, 28.58; HRMS (ESI) $\mathrm{m} / z$ calcd. for $\mathrm{C}_{24} \mathrm{H}_{27} \mathrm{~N}_{4} \mathrm{O}_{3}{ }^{+}[\mathrm{M}+\mathrm{H}]^{+}$: 419.2078; found: 419.2077.
tert-Butyl((1-(dimethylcarbamoyl)-6-methyl-1 H-indol-2-yl) (phenyl)methyl)carbamate (3h)

Following general procedure: $70.8 \mathrm{mg}, 87 \%$ yield; white solid; ${ }^{1} \mathrm{H}$ NMR $\left(500 \mathrm{MHz}, \mathrm{CDCl}_{3}\right) \delta 7.46(\mathrm{~s}, 1 \mathrm{H}$, Ar-H), 7.41-7.35 (m, 4H, Ar-H, N-H), 7.32-7.30 (m, 2H, Ar-H), 7.00 (d, 1H, J 10.0 Hz, Ar-H), 6.87 (s, 1H, Ar-H), $6.17(\mathrm{~s}, 1 \mathrm{H}), 5.21(\mathrm{~s}, 1 \mathrm{H}), 3.05\left(\mathrm{~s}, 6 \mathrm{H}, \mathrm{N}\left(\mathrm{CH}_{3}\right)_{2}\right), 2.48$ (s, $3 \mathrm{H}), 1.48\left(\mathrm{~s}, 9 \mathrm{H}, \mathrm{C}\left(\mathrm{CH}_{3}\right)_{3}\right) ;{ }^{13} \mathrm{C} \mathrm{NMR}\left(126 \mathrm{MHz}, \mathrm{CDCl}_{3}\right)$ $\delta 155.13,155.01,141.04,136.74,134.12,128.55,127.46$, $126.95,125.38,124.25,123.44,119.46,113.74,79.78$, 51.21, 38.42, 28.40, 21.84; HRMS (ESI) $\mathrm{m} / \mathrm{z}$ calcd. for $\mathrm{C}_{24} \mathrm{H}_{30} \mathrm{~N}_{3} \mathrm{O}_{3}^{+}[\mathrm{M}+\mathrm{H}]^{+}:$408.2282; found: 408.2279.

tert-Butyl((6-chloro-1-(dimethylcarbamoyl)-1 H-indol-2-yl) (phenyl)methyl)carbamate (3i)

Following general procedure: $63.2 \mathrm{mg}, 74 \%$ yield; white solid; ${ }^{1} \mathrm{H}$ NMR $\left(500 \mathrm{MHz}, \mathrm{CDCl}_{3}\right) \delta 7.57$ (d, $1 \mathrm{H}$, $J 10.0 \mathrm{~Hz}, \mathrm{Ar}-\mathrm{H}), 7.43$ (s, 1H, Ar-H), 7.39-7.38 (m, 4H, Ar-H, N-H), 7.36-7.32 (m, 1H, Ar-H), 7.27 (dd, 1H, J 8.8, $1.9 \mathrm{~Hz}, \mathrm{Ar}-\mathrm{H}), 6.96$ (s, 1H, Ar-H), 6.16 (s, 1H), 5.18 (s, $1 \mathrm{H}), 3.05\left(\mathrm{~s}, 6 \mathrm{H}, \mathrm{N}\left(\mathrm{CH}_{3}\right)_{2}\right), 1.48\left(\mathrm{~s}, 9 \mathrm{H}, \mathrm{C}\left(\mathrm{CH}_{3}\right)_{3}\right) ;{ }^{13} \mathrm{C} \mathrm{NMR}$ $\left(126 \mathrm{MHz}, \mathrm{CDCl}_{3}\right) \delta 155.08,154.44,134.72,128.73$, $127.75,127.59,126.93,126.01,124.34,120.47,119.50$, 114.74, 80.07, 51.21, 38.45, 28.39; HRMS (ESI) $\mathrm{m} / \mathrm{z}$ calcd. for $\mathrm{C}_{23} \mathrm{H}_{27} \mathrm{ClN}_{3} \mathrm{O}_{3}{ }^{+}[\mathrm{M}+\mathrm{H}]^{+}:$428.1736; found: 428.1733 .

tert-Butyl((1-(dimethylcarbamoyl)-1H-indol-2-yl)( $p$-tolyl) methyl)carbamate (3j)

Following general procedure: $68.4 \mathrm{mg}, 84 \%$ yield; white solid; ${ }^{1} \mathrm{H}$ NMR $\left(500 \mathrm{MHz}, \mathrm{CDCl}_{3}\right) \delta 7.60(\mathrm{~d}, 1 \mathrm{H}, J 10.0 \mathrm{~Hz}$, Ar-H), 7.43 (d, 1H, J 10.0 Hz, Ar-H), 7.30-7.25 (m, 3H, Ar-H, N-H), 7.16-7.13 (m, 3H, Ar-H), 6.95 (s, 1H, Ar-H), $6.14(\mathrm{~s}, 1 \mathrm{H}), 5.16(\mathrm{~s}, 1 \mathrm{H}), 3.04\left(\mathrm{~s}, 6 \mathrm{H}, \mathrm{N}\left(\mathrm{CH}_{3}\right)_{2}\right), 2.35(\mathrm{~s}, 3 \mathrm{H}$, $\left.\mathrm{CH}_{3}\right), 1.46\left(\mathrm{~s}, 9 \mathrm{H}, \mathrm{C}\left(\mathrm{CH}_{3}\right)_{3}\right) ;{ }^{13} \mathrm{C} \mathrm{NMR}\left(126 \mathrm{MHz}, \mathrm{CDCl}_{3}\right)$ $\delta 155.11,154.92,137.13,136.28,129.28,127.66,126.88$, $124.83,123.95,121.78,121.04,120.99,119.94,113.59$, 100.23, 79.91, 50.99, 38.45, 28.41, 21.13; HRMS (ESI) $\mathrm{m} / \mathrm{z}$ calcd. for $\mathrm{C}_{24} \mathrm{H}_{30} \mathrm{~N}_{3} \mathrm{O}_{3}^{+}[\mathrm{M}+\mathrm{H}]^{+}$: 408.2282; found: 408.2282.

tert-Butyl((4-chlorophenyl)(1-(dimethylcarbamoyl)-1 $H$-indol2-yl)methyl)carbamate (3k)

Following general procedure: $72.6 \mathrm{mg}, 85 \%$ yield; white solid; ${ }^{1} \mathrm{H}$ NMR (400 MHz, $\left.\mathrm{CDCl}_{3}\right) \delta 7.60(\mathrm{~d}, 1 \mathrm{H}$, $J 8.0 \mathrm{~Hz}, \mathrm{Ar}-\mathrm{H}), 7.42$ (d, 1H, J 8.0 Hz, Ar-H), 7.32-7.28 (m, 5H, Ar-H, N-H), 7.17 (t, 1H, J $8.0 \mathrm{~Hz}$, Ar-H), 6.91 (s, 1H, Ar-H), 6.15 (s, 1H), $5.14(\mathrm{~s}, 1 \mathrm{H}), 3.04(\mathrm{~s}, 6 \mathrm{H}$, $\left.\mathrm{N}\left(\mathrm{CH}_{3}\right)_{2}\right), 1.45\left(\mathrm{~s}, 9 \mathrm{H}, \mathrm{C}\left(\mathrm{CH}_{3}\right)_{3}\right) ;{ }^{13} \mathrm{C} \mathrm{NMR}(101 \mathrm{MHz}$, $\left.\mathrm{CDCl}_{3}\right) \delta 155.26,154.94,139.98,136.46,133.44,128.94$, 128.52, 127.58, 125.17, 124.37, 122.14, 120.46, 119.94, 113.86, 80.27, 51.17, 38.64, 28.59; HRMS (ESI) $\mathrm{m} / z$ calcd. for $\mathrm{C}_{23} \mathrm{H}_{27} \mathrm{~N}_{3} \mathrm{O}_{3} \mathrm{Cl}^{+}[\mathrm{M}+\mathrm{H}]^{+}$: 428.1736; found: 428.1736 . 
tert-Butyl((1-(dimethylcarbamoyl)- $1 \mathrm{H}$-indol-2-yl) (4-fluorophenyl)methyl)carbamate (3I)

Following general procedure: $71.5 \mathrm{mg}, 87 \%$ yield; white solid; ${ }^{1} \mathrm{H}$ NMR $\left(400 \mathrm{MHz}, \mathrm{CDCl}_{3}\right) \delta 7.62(\mathrm{~d}, 1 \mathrm{H}$, $J$ 6.6 Hz, Ar-H), 7.43 (d, 1H, J 6.1 Hz, Ar-H), 7.39-7.36 (m, 2H, Ar-H), 7.34-7.30 (m, 1H, Ar-H), 7.17-7.20 (m, 1H, Ar-H), 7.06 (t, 2H, J $6.2 \mathrm{~Hz}, \mathrm{Ar}-\mathrm{H}), 6.95$ (s, 1H, Ar-H), $6.17(\mathrm{~s}, 1 \mathrm{H}), 5.20(\mathrm{~s}, 1 \mathrm{H}), 3.06\left(\mathrm{~s}, 6 \mathrm{H}, \mathrm{N}\left(\mathrm{CH}_{3}\right)_{2}\right), 1.48(\mathrm{~s}$, 9H, $\left.\mathrm{C}\left(\mathrm{CH}_{3}\right)_{3}\right) ;{ }^{13} \mathrm{C}$ NMR $\left(100 \mathrm{MHz}, \mathrm{CDCl}_{3}\right) \delta 163.10$, $161.15,155.06,154.78,136.25,130.92,128.52,127.41$, $124.91,124.12,121.89,120.58,119.79,115.52,113.64$, 80.00, 50.86, 38.45, 28.38; HRMS (ESI) $\mathrm{m} / \mathrm{z}$ calcd. for $\mathrm{C}_{23} \mathrm{H}_{27} \mathrm{FN}_{3} \mathrm{O}_{3}{ }^{+}[\mathrm{M}+\mathrm{H}]^{+}:$412.2031; found: 412.2023 .

tert-Butyl((4-cyanophenyl)(1-(dimethylcarbamoyl)-1H-indol2-yl)methyl)carbamate (3m)

Following general procedure: $75.2 \mathrm{mg}$, 90\% yield; white solid; ${ }^{1} \mathrm{H}$ NMR $\left(400 \mathrm{MHz}, \mathrm{CDCl}_{3}\right) \delta 7.61(\mathrm{~d}, 2 \mathrm{H}$, $J 8.0 \mathrm{~Hz}, \mathrm{Ar}-\mathrm{H}), 7.57(\mathrm{~d}, 1 \mathrm{H}, J 8.0 \mathrm{~Hz}, \mathrm{Ar}-\mathrm{H}), 7.50(\mathrm{~d}, 2 \mathrm{H}$, $J 8.0 \mathrm{~Hz}, \mathrm{Ar}-\mathrm{H}), 7.41$ (d, 1H, J 8.0 Hz, Ar-H), 7.32-7.28 (m, 1H, Ar-H), 7.17 (t, 1H, J 8.0 Hz, Ar-H), 6.89 (s, 1H, Ar-H), $6.19(\mathrm{~s}, 1 \mathrm{H}), 5.37(\mathrm{~s}, 1 \mathrm{H}), 3.01\left(\mathrm{~s}, 6 \mathrm{H}, \mathrm{N}\left(\mathrm{CH}_{3}\right)_{2}\right), 1.44$ (s, 9H, $\left.\mathrm{C}\left(\mathrm{CH}_{3}\right)_{3}\right) ;{ }^{13} \mathrm{C} \mathrm{NMR}\left(101 \mathrm{MHz}, \mathrm{CDCl}_{3}\right) \delta 158.08$, 157.50, 149.73, 139.07, 135.39, 130.59, 130.09, 128.09, 127.33, 125.07, 122.47, 122.25, 121.72, 116.67, 114.25, 83.29, 54.22, 41.39, 31.33; HRMS (ESI) $\mathrm{m} / z$ calcd. for $\mathrm{C}_{24} \mathrm{H}_{27} \mathrm{~N}_{4} \mathrm{O}_{3}{ }^{+}[\mathrm{M}+\mathrm{H}]^{+}$: 419.2078 ; found: 419.2075 .

tert-Butyl((1-(dimethylcarbamoyl)-1H-indol-2-yl)(o-tolyl) methyl)carbamate (3n)

Following general procedure: $70.8 \mathrm{mg}, 87 \%$ yield; white solid; ${ }^{1} \mathrm{H}$ NMR $\left(500 \mathrm{MHz}, \mathrm{CDCl}_{3}\right) \delta 7.63$ (d, $J 8.2 \mathrm{~Hz}, 1 \mathrm{H}), 7.54-7.53(\mathrm{~m}, 1 \mathrm{H}, \mathrm{Ar}-\mathrm{H}), 7.34-7.31$ (m, 2H, Ar-H), 7.24-7.20 (m, 4H, Ar-H), 6.78 (s, 1H, Ar-H), 6.38 (s, $1 \mathrm{H}, \mathrm{Ar}-\mathrm{H}), 5.19(\mathrm{~s}, 1 \mathrm{H}), 3.02\left(\mathrm{~s}, 6 \mathrm{H}, \mathrm{N}\left(\mathrm{CH}_{3}\right)_{2}\right), 2.36(\mathrm{~s}, 3 \mathrm{H}$, $\left.\mathrm{CH}_{3}\right), 1.49\left(\mathrm{~s}, 9 \mathrm{H}, \mathrm{C}\left(\mathrm{CH}_{3}\right)_{3}\right) ;{ }^{13} \mathrm{C} \mathrm{NMR}\left(126 \mathrm{MHz}, \mathrm{CDCl}_{3}\right)$ $\delta$ 155.01, 154.83, 139.17, 136.27, 135.77, 130.67, 127.81, $127.43,126.17,125.83,125.04,124.04,121.85,120.46$, 119.80, 113.57, 79.74, 48.22, 38.40, 28.41, 19.17; HRMS (ESI) $\mathrm{m} / z$ calcd. for $\mathrm{C}_{24} \mathrm{H}_{30} \mathrm{~N}_{3} \mathrm{O}_{3}{ }^{+}[\mathrm{M}+\mathrm{H}]^{+}$: 408.2282; found: 408.2279 .

tert-Butyl((2-bromophenyl)(1-(dimethylcarbamoyl)- $1 \mathrm{H}$-indol2-yl)methyl)carbamate (30)

Following general procedure: $76.3 \mathrm{mg}, 81 \%$ yield; white solid; ${ }^{1} \mathrm{H}$ NMR (500 MHz, $\left.\mathrm{CDCl}_{3}\right) \delta$ 7.64-7.60 (m, $3 \mathrm{H}, \mathrm{Ar}-\mathrm{H}), 7.50$ (d, $1 \mathrm{H}, J 5.0 \mathrm{~Hz}, \mathrm{Ar}-\mathrm{H}), 7.39-7.32$ (m, $2 \mathrm{H}$, Ar-H), 7.25-7.19 (m, 2H, Ar-H), 6.78 (s, 1H, Ar-H), 6.48 (s, 1H, Ar-H), $5.27(\mathrm{~s}, 1 \mathrm{H}), 3.03\left(\mathrm{~s}, 6 \mathrm{H}, \mathrm{N}\left(\mathrm{CH}_{3}\right)_{2}\right), 1.49$ (s, 9H, $\left.\mathrm{C}\left(\mathrm{CH}_{3}\right)_{3}\right) ;{ }^{13} \mathrm{C} \mathrm{NMR}\left(126 \mathrm{MHz}, \mathrm{CDCl}_{3}\right) \delta 154.89$, $154.75,136.18,133.33,129.05,127.97,127.65,125.24$,
124.11, 121.93, 119.72, 113.59, 79.99, 51.37, 38.44, 28.38; HRMS (ESI) $m / z$ calcd. for $\mathrm{C}_{23} \mathrm{H}_{27} \mathrm{~N}_{3} \mathrm{O}_{3}{ }^{+}[\mathrm{M}+\mathrm{H}]^{+}$: 472.1231; found 472.1231 .

tert-Butyl((1-(dimethylcarbamoyl)-1H-indol-2-yl) (naphthalen-1-yl)methyl)carbamate (3p)

Following general procedure: $68.2 \mathrm{mg}, 77 \%$ yield; white solid; ${ }^{1} \mathrm{H}$ NMR $\left(400 \mathrm{MHz}, \mathrm{CDCl}_{3}\right) \delta 8.04(\mathrm{~d}, 1 \mathrm{H}$, $J$ 8.0 Hz, Ar-H), 7.89-7.81 (m, 2H, Ar-H), $7.64(\mathrm{~d}, 1 \mathrm{H}$, $J$ 8.2 Hz, Ar-H), 7.51-7.44 (m, 5H, Ar-H), 7.32 (t, 1H, $J 8.0 \mathrm{~Hz}, \mathrm{Ar}-\mathrm{H}), 7.18$ (t, 1H, J 7.5 Hz, Ar-H), $6.96(\mathrm{~d}, 1 \mathrm{H}$, J 7.6 Hz, Ar-H), 6.80 (s, 1H, Ar-H), 5.27 (s, 1H), 2.93 (s, $\left.6 \mathrm{H}, \mathrm{N}\left(\mathrm{CH}_{3}\right)_{2}\right), 1.48\left(\mathrm{~s}, 9 \mathrm{H}, \mathrm{C}\left(\mathrm{CH}_{3}\right)_{3}\right) ;{ }^{13} \mathrm{C} \mathrm{NMR}(101 \mathrm{MHz}$, $\left.\mathrm{CDCl}_{3}\right) \delta 158.02,157.77,139.26,136.92,133.97,131.70$, $131.39,130.72,129.28,128.70,128.36,128.29,127.04$, 126.76, 124.87, 123.67, 122.87, 116.61, 82.87, 51.20, 41.34, 31.39; HRMS (ESI) m/z calcd. for $\mathrm{C}_{27} \mathrm{H}_{30} \mathrm{~N}_{3} \mathrm{O}_{3}{ }^{+}$ $[\mathrm{M}+\mathrm{H}]^{+}:$444.2282; found: 444.2284 .

tert-Butyl((1-(dimethylcarbamoyl)-1H-indol-2-yl)(thiophen2-yl)methyl)carbamate (3q)

Following general procedure: $57.4 \mathrm{mg}, 72 \%$ yield; white solid; ${ }^{1} \mathrm{H}$ NMR $\left(400 \mathrm{MHz}, \mathrm{CDCl}_{3}\right) \delta 7.63(\mathrm{~d}, 1 \mathrm{H}$, $J 8.0 \mathrm{~Hz}, \mathrm{Ar}-\mathrm{H}), 7.47$ (d, 1H, J 8.0 Hz, Ar-H), 7.32-7.23 (m, 2H, Ar-H, N-H), 7.20-7.15 (m, 2H, Ar-H), 6.98-6.96 (m, 2H, Ar-H), 6.41 (s, 1H, Ar-H), $5.24(\mathrm{~s}, 1 \mathrm{H}), 3.07$ (s, $\left.6 \mathrm{H}, \mathrm{N}\left(\mathrm{CH}_{3}\right)_{2}\right), 1.46\left(\mathrm{~s}, 9 \mathrm{H}, \mathrm{C}\left(\mathrm{CH}_{3}\right)_{3}\right) ;{ }^{13} \mathrm{C} \mathrm{NMR}(100 \mathrm{MHz}$, $\left.\mathrm{CDCl}_{3}\right) \delta 155.08,155.02,145.65,136.46,127.50,127.06$, 125.34, 124.86, 124.77, 124.28, 122.09, 120.49, 120.01, 113.91, 80.32, 47.75, 38.68, 28.59; HRMS (ESI) $\mathrm{m} / \mathrm{z}$ calcd. for $\mathrm{C}_{21} \mathrm{H}_{26} \mathrm{~N}_{3} \mathrm{O}_{3} \mathrm{~S}^{+}[\mathrm{M}+\mathrm{H}]^{+}$: 400.1690; found: 400.1689 .

tert-Butyl(1-(1-(dimethylcarbamoyl)- $1 \mathrm{H}$-indol-2-yl)2-ethylbutyl)carbamate (3r)

Following general procedure: $52.6 \mathrm{mg}, 68 \%$ yield; white solid; ' ${ }^{\mathrm{H}} \mathrm{NMR}\left(400 \mathrm{MHz}, \mathrm{CDCl}_{3}\right) \delta$ 7.65-7.59 (m, 2H), 7.31-7.29 (m, 1H, Ar-H), 7.21-7.17 (m, 2H, Ar-H), $5.04(\mathrm{~s}, 1 \mathrm{H}), 4.78(\mathrm{~s}, 1 \mathrm{H}), 3.08\left(\mathrm{~s}, 6 \mathrm{H}, \mathrm{N}\left(\mathrm{CH}_{3}\right)_{2}\right), 1.81$ (s, $1 \mathrm{H}), 1.57\left(\mathrm{~m}, 2 \mathrm{H}, \mathrm{CH}_{2}\right), 1.42-1.38\left(\mathrm{~m}, 11 \mathrm{H}, \mathrm{C}\left(\mathrm{CH}_{3}\right)_{3}, \mathrm{CH}_{2}\right)$, 0.97 (t, $J 8.0 \mathrm{~Hz}, 3 \mathrm{H}, \mathrm{CH}_{3}$ ), 0.89 (t, $J 8.0 \mathrm{~Hz}, 3 \mathrm{H}, \mathrm{CH}_{3}$ ); ${ }^{13} \mathrm{C}$ NMR $\left(100 \mathrm{MHz}, \mathrm{CDCl}_{3}\right) \delta 155.77,155.32,136.36$, $128.17,123.97,123.54,121.81,120.65,119.87,113.92$, 79.65, 49.73, 45.21, 38.70, 29.91, 28.62, 22.87, 21.85, 11.81; HRMS (ESI) $m / z$ calcd for. $\mathrm{C}_{22} \mathrm{H}_{34} \mathrm{~N}_{3} \mathrm{O}_{3}{ }^{+}[\mathrm{M}+\mathrm{H}]^{+}$: 388.2595; found: 388.2596 .

\section{Supplementary Information}

Supplementary information (NMR spectra of new compounds) is available free of charge at http://jbcs.sbq. org.br as PDF file. 


\section{Acknowledgments}

We gratefully acknowledge financial support from Natural Science Fund for Colleges and Universities in Jiangsu Province (15KJB150004), and Nature Science Foundation of Changzhou Institute of Technology (No. YN1416).

\section{References}

1. Colby, D. A.; Bergman, R. G.; Ellman, J. A.; Chem. Rev. 2010, 110, 624; Song, G.; Wang, F.; Li, X.; Chem. Soc. Rev. 2012, 41, 3651; Zhang, B.; Li, B.; Zhang, X.; Fan, X.; Org. Lett. 2017, 19, 2294; Chen, X.; Yang, S.; Li, H.; Wang, B.; Song, G.; ACS Catal. 2017, 7, 2392; Cai, S.; Lin, S.; Yi, X.; Xi, C.; J. Org. Chem. 2017, 82, 512; Li, Y.; Wang, Q.; Yang, X.; Xie, F.; Li, X.; Org. Lett. 2017, 19, 3410; Satoh, T.; Miura, M.; Chem.-Eur. J. 2010, 16, 11212; Patureau, F. W.; Wencel-Delord, J.; Glorius, F.; Aldrichimica Acta 2012, 45, 31.

2. Colby, D. A.; Tsai, A. S.; Bergman, R. G.; Ellman, J. A.; Acc. Chem. Res. 2012, 45, 814; Kuhl, N.; Schröder, N.; Glorius, F.; Adv. Synth. Catal. 2014, 356, 1443; Hyster, T. K.; Dalton, D. M.; Rovis, T.; Chem. Sci. 2015, 6, 254; Michailidis, F. R.; Sedillo, K. F.; Neely, J. M.; Rovis, T.; J. Am. Chem. Soc. 2015, 137, 8892; Jia, J. L.; Shi, J. J.; Zhou, J.; Liu, X. L.; Song, Y. L.; Xu, E.; Yi, W.; Chem. Commun. 2015, 51, 2925; Lu, M. Z.; Lu, P.; Xu, Y. H.; Loh, T.-P.; Org. Lett. 2014, 16, 2614.

3. Tsai, A. S.; Tauchert, M. E.; Bergman, R. G.; Ellman, J. A.; J. Am. Chem. Soc. 2011, 133, 1248; Li, X.; Yu, S.; Wang, F.; Wan, B.; Yu, X.; Angew. Chem., Int. Ed. 2013, 52, 2577; Zhu, C.; Falck, J. R.; Chem. Commun. 2012, 48, 1674; Wu, X.; Wang, B.; Zhou, Y.; Liu, H.; Org. Lett. 2017, 19, 12944.

4. Arunotayanun, W.; Gibbons, S.; Nat. Prod. Rep. 2012, 29, 1304; Ishikura, M.; Yamada, K.; Abe, T.; Nat. Prod. Rep. 2010, 27, 1630; Lavrado, J.; Moreira, R.; Paulo, A.; Curr. Med. Chem. 2010, 17, 2348.

5. Bosch, J.; Bennasar, M. L.; Synlett 1995, 587.

6. Faulkner, D. J.; Nat. Prod. Rep. 2002, 19, 1.
7. Agarwal, S.; Caemmerer, S.; Filali, S.; Froehner, W.; Curr. Org. Chem. 2005, 9, 1601.

8. O'Connor, S. E.; Maresh, J. J.; Nat. Prod. Rep. 2006, 23, 532.

9. Zhou, H.; Wang, J.; Yao, B.; Wong, S.; Djakovic, S.; Kumar, B.; Rice, J.; Valle, E.; Soriano, F.; Menon, M. K.; Madriaga, A.; Soly, S. K.; Kumar, A.; Parlati, F.; Yakes, F. M.; Shawver, L.; Moigne, R. L.; Anderson, D. J.; Rolfe, M.; Wustrow, D.; J. Med. Chem. 2015, 58, 9480.

10. Zhou, B.; Yang, Y.; Lin, S.; Li, Y.; Adv. Synth. Catal. 2013, 355, 360.

11. Li, Y.; Li, B.; Wang, W.; Huang, W.; Zhang, X.; Chen, K.; Shi, Z.; Angew. Chem., Int. Ed. 2011, 123, 2163; Liu, X.; Li, X.; Liu, H.; Guo, Q.; Lan, J.; Wang, R.; You, J.; Org. Lett. 2015, 17, 2936; Wang, H.; Yu, S.; Qi, Z.; Li, X.; Org. Lett. 2015, 17, 2812; Feng, C.; Loh, T.-P.; Angew. Chem., Int. Ed. 2014, 53, 2722; Zhou, B.; Du, J.; Yang, Y.; Li, Y.; Chem.-Eur. J. 2014, 20, 12768; Liu, B.; Hu, P.; Zhou, X.; Bai, D.; Chang, J.; Li, X.; Org. Lett. 2017, 19, 2086.

12. Chen, J.; Song, G.; Pan, C.-L.; Li, X.; Org. Lett. 2010, 12, 5426; Huestis, M. P.; Chan, L. N.; Stuart, D. R.; Fagnou, K.; Angew. Chem., Int. Ed. 2011, 50, 1338; Hoshino, Y.; Shibata, Y.; Tanaka, K.; Adv. Synth. Catal. 2014, 356, 1577; Shibata, Y.; Tanaka, K.; Angew. Chem., Int. Ed. 2011, 50, 10917; Ackermann, L.; Lygin, A. V.; Org. Lett. 2012, 14, 764.

13. Schipper, D. J.; Hutchinson, M.; Fagnou, K.; J. Am. Chem. Soc. 2010, 132, 6910.

14. Kanazawa, A. M.; Denis, J.; Greene, A. E.; J. Org. Chem. 1994, 59, 1238.

15. Wenzel, A. G.; Jacobsen, E. N.; J. Am. Chem. Soc. 2002, 124, 12964.

16. Song, J.; Wang, Y.; Deng, L.; J. Am. Chem. Soc. 2006, 128, 6048.

17. Gizecki, P.; Ait Youcef, R.; Poulard, C.; Dhal, R.; Dujardin, G.; Tetrahedron Lett. 2004, 45, 9589.

18. Rampalakos, C.; Wulff, W. D.; Adv. Synth. Catal. 2008, 350, 1785.

Submitted: September 18, 2017

Published online: November 27, 2017 